\title{
Effects of secondary
}

\section{hyperparathyroidism treatment on} hemoglobin levels and erythropoietin doses in hemodialysis patients

\author{
Carlo Massimetti ${ }^{1 *}$, Antonio Bellasi ${ }^{2}$ and Sandro Feriozzi ${ }^{1}$ \\ ${ }^{1}$ Nephrology and Dialysis Unit, Belcolle Hospital, Viterbo, Italy \\ ${ }^{2}$ Department of Research, Innovation, Brand Reputation, Ospedale di Bergamo, ASST Papa \\ Giovanni XXIII Hospital, Bergamo, Italy
}

\section{Abstract}

Aim: Secondary hyperparathyroidism (SHPT) is an often underestimated cause of anemia in hemodialysis (HD) patients. The aim of this study was to assess the effect of pharmacological correction of SHPT on anemia and erythropoiesis-stimulating agents (ESAs) need.

Methods: For the purpose of this retrospective pre-post observational study, we selected 55 HD patients, receiving HD at one single center, in the period from January 2005 to December 2020. The follow-up (F-U) lasted 12 months. The selection criteria were parathormone (PTH) levels > $300 \mathrm{pg} / \mathrm{mL}$, and hemoglobin $(\mathrm{Hb})$ levels $<11 \mathrm{~g} / \mathrm{dL}$, despite treatment with ESAs. Parametric and non-parametric tests were used when appropriate. In the light of exploratory nature of the study, the limited sample size and in consideration of the pre-post-design, no further adjustment for potential confounders is performed.

Results: The hemoglobin levels throughout the study were correlated to serum PTH $(r=-.257$, $p<0.01$ ). At the end of the $\mathrm{F}-\mathrm{U}$, in the 40 patients whose $\mathrm{PTH}$ levels decreased $\geq 30 \%$ (responders group) $\mathrm{Hb}$ levels increased from $10.3 \pm 0.5 \mathrm{~g} / \mathrm{dL}$ to $12.2 \pm 1.1 \mathrm{~g} / \mathrm{dL}(p<0.001)$, and ESAs doses decreased from $141 \pm 101 \mathrm{lU} / \mathrm{kg} / \mathrm{b}$.w. $/$ week to $94 \pm 76 \mathrm{IU} / \mathrm{kg} / \mathrm{b}$.w. $/$ week $(p<0.05)$. On the contrary, in the non-responders group $\mathrm{Hb}$ levels did not change $10.3 \pm 0.5 \mathrm{gr} / \mathrm{dL}$ at baseline and $10.1 \pm 1.1$ $\mathrm{gr} / \mathrm{dL}$ at $\mathrm{F}-\mathrm{U}(\mathrm{P}=\mathrm{NS})$, and the mean doses of ESAs increased from $144 \pm 75 \mathrm{lU} / \mathrm{kg} / \mathrm{b} . \mathrm{w}$./week to $218 \pm 145 \mathrm{IU} / \mathrm{kg} / \mathrm{b} . w . /$ week $(P=\mathrm{NS})$.

Conclusion: Adequate control of SHPT is associated with concomitant improvement of anemia and decrease in ESAs need. Future endeavors are required to confirm these preliminary results.

\section{Introduction}

Anemia is a common complication in HD patients, causing considerable morbidity and dramatically reducing their quality of life [1]. Anemia in HD patients recognizes various causes, but the most important is a deficiency in erythropoietin (EPO) [2]. Erythropoiesis-stimulating agents corrects anemia in the majority of the patients, but responsiveness to ESAs varies widely among HD patients [3]. The patients with a reduced hematopoietic response to ESAs are called ESA resistant or hyporesponsive [4]. The hyporesponsiveness to ESAs has been related to many factors: iron deficiency, inflammatory state, older age,

\section{More Information}

*Address for Correspondence: Carlo Massimetti, Centro di Riferimento di Nefrologia e Dialisi, Ospedale Belcolle, Via Sammartinese,snc, 01100 Viterbo, Italy, Email: cmassimetti@libero.it

Submitted: May 14, 2021

Approved: October 14, 2021

Published: October 15, 2021

How to cite this article: Massimetti C, Bellasi A, Feriozzi S. Effects of secondary hyperparathyroidism treatment on hemoglobin levels and erythropoietin doses in hemodialysis patients. J Clini Nephrol. 2021; 5: 088-094.

DOI: 10.29328/journal.jcn.1001081

Copyright: (c) 2021 Massimetti C, et al. This is an open access article distributed under the Creative Commons Attribution License, which permits unrestricted use, distribution, and reproduction in any medium, provided the original work is properly cited.

Keywords: Anemia; Erythropoietin Erythropoiesis-stimulating agents; Parathormone; Secondary hyperparathyroidism

Check for updates OPEN ACCESS infectious and neoplastic diseases [5]. However, one the less recognized reasons of anemia and hyporesponsiveness to ESAs is parathyroid hormone (PTH) over secretion, which is a very common co-morbid situation among HD patients. Pathogenesis of anemia and reduced hematopoietic response to ESAs in SHPT is still unclear. There are various hypotheses such as a direct effect of PTH on endogenous EPO synthesis, on bone marrow erythroid progenitor cells, and on red blood cell survival $[6,7]$. An indirect effect through the induction of bone marrow fibrosis also has been proposed [8]. Secondary hyperparathyroidism is associated with an increase in fibroblast growth factor 23 (FGF23) levels which has been demonstrated negatively regulates erythropoiesis 
through suppression of EPO production and EPO receptor expression [9]. Clinical studies have shown that elevated PTH levels were independently associated with ESAs hyporesponsiveness and that the treatment of SHPT using either vitamin D receptor activators (VDRAs), calcimimetics, or parathyroidectomy (PTx) leads to improvement of anemia [10-13]. Therefore, we wanted to evaluate in a group of patients receiving maintenance dialysis if a stringent control of SHPT is associated with an improvement in anemia and a reduction in the ESAs need. Specifically, we herein describe the main results of a retrospective, monocenter, pre-poststudy aimed at testing wheter PTH lowering below 300 pg/ $\mathrm{mL}$ is associated with a concomitant increase in Hb levels and/or reduction in ESAs weekly dosage.

\section{Materials and methods}

We retrospectively selected 55 out of 299 patients undergoing HD treatment at one single center (Belcolle Hospital, Viterbo, Italy) between January 2005 and December 2020 fulfilling the inclusion and exclusion criteria. The study was notified to the ethical committee of Belcolle Hospital, Viterbo. All selected patients were required to have SHPT defined according to National Kidney Foundation (NKF) Kidney Disease Outcomes Quality Initiative (K/DOQI) clinical practice guidelines for bone and mineral metabolism [14] as PTH levels greater than $300 \mathrm{pg} / \mathrm{ml}$ and anemia defined as $\mathrm{Hb}$ levels $<11 \mathrm{~g} / \mathrm{dL}$ irrespective of treatment with ESAs. Other inclusion criteria in the study were: a dialysis vintage greater than six months at the time of enrollment; an observation period of at least 12 months; age > years 18; determination of transferrin saturation (TSAT) and ferritin levels at least every six months; baseline TSAT $>20 \%$, and serum ferritin levels > $100 \mathrm{ng} / \mathrm{mL}$. Exclusion criteria were: hematological disorders; bleeding in the last six months; malignancies; treatment with immunosuppressive drugs; infectious; chronic inflammatory pathology; autoimmune pathologies; request for blood transfusions. All patients were treated with paricalcitol and/ or calcimimetics (cinacalcet or etelcalcetide). According to the KDIGO Clinical Practice Guideline for the Diagnosis, Evaluation, Prevention, and Treatment of Chronic Kidney Disease-Mineral and Bone Disorder (CKD-MBD) the type of treatment for the SHPT was decided on the basis of the serum calcium, phosphorus, and PTH levels [15]. During therapy with calcimimetics when serum calcium was reduced to values lower than $8.5 \mathrm{mg} / \mathrm{dL}$, paricalcitol and subsequently calcium carbonate were introduced at dosages not greater than $15 \mu \mathrm{g}$ per week and $1.5 \mathrm{~g} /$ day, respectively. As suggested by the Clinical practice guidelines, Kidney Disease: Improving Global Outcomes (KDIGO) 2012, during the study ESAs treatment was associated with the concomitant administration of intravenous iron to maintain TSAT $>20 \%$ and ferritin levels $>100 \mathrm{ng} / \mathrm{mL}$ [16]. In presence of Hb levels $>13 \mathrm{~g} / \mathrm{dL}$, ESAs doses were halved then if $\mathrm{Hb}$ persistently > $13 \mathrm{~g} / \mathrm{dL}$ suspended.
The primary end point of the study was the reduction in PTH levels $\geq 30 \%$ and if it was associated with an improvement in $\mathrm{Hb}$ levels. The secondary end point was represented by changes in ESAs doses. Collected laboratory data included serum levels of total calcium, phosphate, PTH, total alkaline phosphatase ( $\mathrm{t}-\mathrm{ALP}$ ), albumin, Hb, TSAT and ferritin. Calcium, phosphate and $\mathrm{Hb}$ levels were determined monthly, while the other parameters were determined at baseline, and then at six and 12 months after starting SHPT treatment. Monthly blood pressure and body weight (mean of the predialysis values of the month), ESAs, phosphate binders, paricalcitol, and calcimimetics doses were also collected for current analysis.

\section{Statistical analysis}

Results were expressed as mean \pm standard deviation $(\mathrm{M} \pm \mathrm{SD})$ for continuous variables.

Parametric and non-parametric tests were used when appropriate to compare between and within groups characteristics. To gauge the association between SHPTH and anemia, we firstinvestigated the correlations between biologic measures at study inception utilizing the Spearman $(\rho)$ rank correlation. Also a stepwise approach was implemented to select the most parsimonious multivariable adjusted linear regression model to predict $\mathrm{Hb}$ levels at baseline and at different time during F-U (Hb values at baseline, six and 12 months, dependent variable). The predictive variables were selected on the basis of the significance of the correlation in the Spearman analysis $(p<0.05)$. These included the following most relevant variables: age, HD vintage, serum phosphorous and PTH levels, t-ALP, ESAs doses, iron intravenous therapy, average doses of paricalcitol and calcimimetics. Subsequently, we stratified the study cohort according to PTH reduction at follow-up as responders (PTH reduction $\geq 30 \%$ ) or non responders (no PTH reduction or reduction $<30 \%$ ) to SHPT treatments. Hemoglobin as well as changes in ESAs doses within and between groups were compared with parametric and non-parametric tests when appropriate. In light of the exploratory nature of the study, the limited sample size and in consideration of the pre-poststudy design, no multivariable adjustment for potential confounders is performed.

For all the statistical tests used a $p$ value $<0.05$ was considered statistically significant. Data were analyzed using the Statistical Package for Social Sciences (SPSS) for Windows, version 15.0 software (SPSS Inc., IL, USA).

\section{Results}

Overall, we analyzed data of 55 middle-age (mean age of $62 \pm 14$ years; 26 males and 14 females) on maintenance HD (mean dialysis vintage: $45 \pm 43$ months). Throughout the study the $\mathrm{Hb}$ levels were significantly correlated to serum PTH and t-ALP levels, as well as to the ESAs doses. Other 
Table 1: Main clinical changes during secondary hyperparathyroidism treatment $(n=55)$.

\begin{tabular}{|c|c|c|c|}
\hline Months & baseline & 6 & 12 \\
\hline Serum calcium, mg/dL & $8.9 \pm 0.6$ & $8.9 \pm 0.5$ & $8.9 \pm 0.6$ \\
\hline Serum phosphorus, mg/dL & $5.7 \pm 1.6$ & $5.1 \pm 1.5^{*}$ & $4.8 \pm 1.4^{\circ}$ \\
\hline Serum PTH, pg/mL & $680 \pm 399$ & $465 \pm 296^{\circ}$ & $435 \pm 385^{\wedge}$ \\
\hline Serum total alkaline phosphatase, $\mathrm{mU} / \mathrm{mL}$ & $110 \pm 58$ & $108 \pm 70$ & $109 \pm 62$ \\
\hline Serum albumin, g/dL & $3.7 \pm 0.4$ & $3.7 \pm 0.2$ & $3.8 \pm 0.3$ \\
\hline Hematocrit, \% & $32 \pm 3$ & $35 \pm 4^{\wedge}$ & $36 \pm 5^{\wedge}$ \\
\hline Hemoglobin, g/dL & $10.3 \pm 0.5$ & $11.5 \pm 1.4^{\wedge}$ & $11.7 \pm 1.5^{\wedge}$ \\
\hline Transferrin saturation, $\%$ & $36 \pm 21$ & $30 \pm 15$ & $35 \pm 20$ \\
\hline Ferritin, $\mathrm{ng} / \mathrm{mL}$ & $872 \pm 932$ & $971 \pm 912$ & $1157 \pm 842$ \\
\hline Paricalcitol, $\mu \mathrm{g} /$ week (\% pts) & $9.3 \pm 4.0(82)$ & $9.2 \pm 3.8(82)$ & $8.3 \pm 4.0(82)$ \\
\hline Cinacalcet, mg/day (\% pts) & $30 \pm 0(20)$ & $32 \pm 8(20)$ & $30 \pm 0(22)$ \\
\hline Etelcalcetide, mg/week (\% pts) & $11.8 \pm 3.8(25)$ & $21.9 \pm 5.5(25)^{\wedge}$ & $26.7 \pm 8.6(25)^{\wedge}$ \\
\hline Sevelamer carbonate, g/day (\% pts) & $3.9 \pm 2.1(74)$ & $3.8 \pm 1.9(74)$ & $3.7 \pm 2.0(78)$ \\
\hline Calcium carbonate, g/day (\% pts) & $1.0 \pm 0.3(27)$ & $0.9 \pm 0.3(38)$ & $1.0 \pm 0.4(42)$ \\
\hline ESAs, IU/kg/b.w./week, (\% pts) & $141 \pm 95(100)$ & $144 \pm 100(100)$ & $129 \pm 114(96)$ \\
\hline Ferric gluconate, mg/week (\% pts) & $117 \pm 53(80)$ & $145 \pm 102(84)$ & $106 \pm 55(82)$ \\
\hline Ferric carboxymaltose, $\mathrm{mg} /$ week (\% pts) & $60 \pm 22(9)$ & $39 \pm 18(13)$ & $48 \pm 36(14)$ \\
\hline Body weight, $\mathrm{Kg}$ & $71 \pm 14$ & $72 \pm 16$ & $71 \pm 15$ \\
\hline
\end{tabular}

$p$ vs. baseline: ${ }^{*}<0.05 ;{ }^{\circ}<0.01 ;{ }^{\wedge}<0.001$. Abbreviations: PTH: Parathormone; ESAs: Erythropoiesis-Stimulating Agents; \%pts: Percentage of Patients treated; b.w: body weight.

Table 2: Main clinical changes during secondary hyperparathyroidism treatment in the responders group $(n=40)$.

\begin{tabular}{|c|c|c|c|}
\hline Months & baseline & 6 & 12 \\
\hline Serum calcium, mg/dL & $8.9 \pm 0.5$ & $8.9 \pm 0.4$ & $9.0 \pm 0.5$ \\
\hline Serum phosphorus, mg/dL & $5.8 \pm 1.7$ & $5.0 \pm 1.6^{*}$ & $4.5 \pm 1.0^{\wedge}$ \\
\hline Serum PTH, pg/mL & $731 \pm 425$ & $430 \pm 290^{\wedge}$ & $309 \pm 177^{\wedge}$ \\
\hline Percent reduction of PTH, \% & & -39 & -56 \\
\hline Serum total alkaline phosphatase, $\mathrm{mU} / \mathrm{mL}$ & $112 \pm 59$ & $105 \pm 55$ & $106 \pm 58$ \\
\hline Serum albumin, g/dL & $3.7 \pm 0.4$ & $3.7 \pm 0.3$ & $3.8 \pm 0.3$ \\
\hline Hematocrit, \% & $32 \pm 2$ & $35 \pm 4^{\wedge}$ & $37 \pm 4^{\wedge}$ \\
\hline Hemoglobin, g/dL & $10.4 \pm 0.5$ & $11.8 \pm 1.4^{\wedge}$ & $12.2 \pm 1.1^{\wedge}$ \\
\hline Transferrin saturation, \% & $35 \pm 18$ & $31 \pm 16$ & $35 \pm 20$ \\
\hline Ferritin, $\mathrm{ng} / \mathrm{mL}$ & $977 \pm 1029$ & $1044 \pm 985$ & $1147 \pm 813$ \\
\hline Paricalcitol, $\mu \mathrm{g} /$ week (\% pts) & $9.7 \pm 4.2(30)$ & $9.7 \pm 3.6(85)$ & $8.3 \pm 3.6(85)$ \\
\hline Cinacalcet, mg/day (\% pts) & $30 \pm 0(17)$ & $30 \pm 0(17)$ & $30 \pm 0(17)$ \\
\hline Etelcalcetide, mg/week (\% pts) & $11.9 \pm 3.8(28)$ & $21.8 \pm 5.9(30)^{\wedge}$ & $26.2 \pm 8.7(30)^{\wedge}$ \\
\hline Sevelamer carbonate, g/day (\% pts) & $3.8 \pm 2.1(77)$ & $3.6 \pm 1.9(77)$ & $3.3 \pm 1.8(77)$ \\
\hline Calcium carbonate, g/day (\% pts) & $1.0 \pm 0.3(22)$ & $1.0 \pm 0.2(35)$ & $1.1 \pm 0.4(40)$ \\
\hline ESAs, IU/kg/b.w./week, (\% pts) & $141 \pm 101(100)$ & $132 \pm 104(100)$ & $94 \pm 76(95)^{*}$ \\
\hline Ferric gluconate, mg/week (\% pts) & $110 \pm 54(82)$ & $100 \pm 52(85)$ & $101 \pm 57(82)$ \\
\hline Ferric carboxymaltose, $\mathrm{mg} / \mathrm{week}$ (\% pts) & $62 \pm 25(10)$ & $42 \pm 16(12)$ & $60 \pm 34(15)$ \\
\hline Body weight, $\mathrm{Kg}$ & $72 \pm 16$ & $72 \pm 16$ & $71 \pm 16$ \\
\hline$p$ vs. basale: ${ }^{*}<0.05 ;^{\circ}<0.01 ;^{\wedge}<0.001$ & & & \\
\hline
\end{tabular}

Table 3: Main clinical changes during secondary hyperparathyroidism treatment in the non-responders group $(n=15)$.

\begin{tabular}{|c|c|c|c|}
\hline Months & baseline & 6 & 12 \\
\hline Serum calcium, mg/dL & $8.9 \pm 0.9$ & $8.9 \pm 0.7$ & $8.7 \pm 0.8$ \\
\hline Serum phosphorus, mg/dL & $5.6 \pm 1.3$ & $5.5 \pm 1.2$ & $5.6 \pm 1.8$ \\
\hline Serum PTH, pg/mL & $543 \pm 290$ & $560 \pm 299$ & $771 \pm 565$ \\
\hline Percent increase of PTH, \% & & 2 & 52 \\
\hline Serum total alkaline phosphatase, $\mathrm{mU} / \mathrm{mL}$ & $108 \pm 56$ & $118 \pm 73$ & $117 \pm 78$ \\
\hline Serum albumin, g/dL & $3.7 \pm 0.5$ & $3.6 \pm 0.4$ & $3.8 \pm 0.3$ \\
\hline Hematocrit, \% & $33 \pm 4$ & $34 \pm 4$ & $32 \pm 3$ \\
\hline Hemoglobin, g/dL & $10.2 \pm 0.5$ & $10.8 \pm 1.3$ & $10.1 \pm 1.1$ \\
\hline Transferrin saturation, $\%$ & $40 \pm 28$ & $30 \pm 15$ & $36 \pm 22$ \\
\hline Ferritin, $\mathrm{ng} / \mathrm{mL}$ & $594 \pm 536$ & $830 \pm 710$ & $1182 \pm 947^{*}$ \\
\hline Paricalcitol, $\mu \mathrm{g} /$ week (\% pts) & $8.2 \pm 3.3(73)$ & $7.7 \pm 4.1(73)$ & $8.2 \pm 5.1(73)$ \\
\hline Cinacalcet, mg/day (\% pts) & $30 \pm 0(27)$ & $30 \pm 0(27)$ & $30 \pm 0(27)$ \\
\hline Etelcalcetide, mg/week (\% pts) & $11.2 \pm 5.3(13)$ & $22.5 \pm 0(13)$ & $22.5 \pm 0(13)$ \\
\hline Sevelamer carbonate, g/day (\% pts) & $4.4 \pm 1.8(67)$ & $4.4 \pm 1.8(67)$ & $4.4 \pm 1.8(67)$ \\
\hline Calcium carbonate, g/day (\% pts) & $0.9 \pm 0.2(40)$ & $0.8 \pm 0.4(47)$ & $0.8 \pm 0.4(47)$ \\
\hline ESAs, IU/kg/b.w./week, (\% pts) & $144 \pm 75(100)$ & $174 \pm 85(100)$ & $218 \pm 145(100)$ \\
\hline Ferric gluconate, $\mathrm{mg} /$ week (\% pts) & $136 \pm 46(73)$ & $120 \pm 49(80)$ & $120 \pm 49(80)$ \\
\hline Ferric carboxymaltose, $\mathrm{mg} /$ week (\% pts) & $50 \pm 0(7)$ & $31 \pm 26(13)$ & $12 \pm 0(13)$ \\
\hline Body weight, $\mathrm{Kg}$ & $71 \pm 15$ & $72 \pm 15$ & $71 \pm 14$ \\
\hline$p$ vs. baseline: ${ }^{*}<0.05$ & & & \\
\hline
\end{tabular}


Table 4: Comparison between the responders $(n=40)$ and non-responders $(n=15)$ group for the main clinical data at baseline and at F-U.

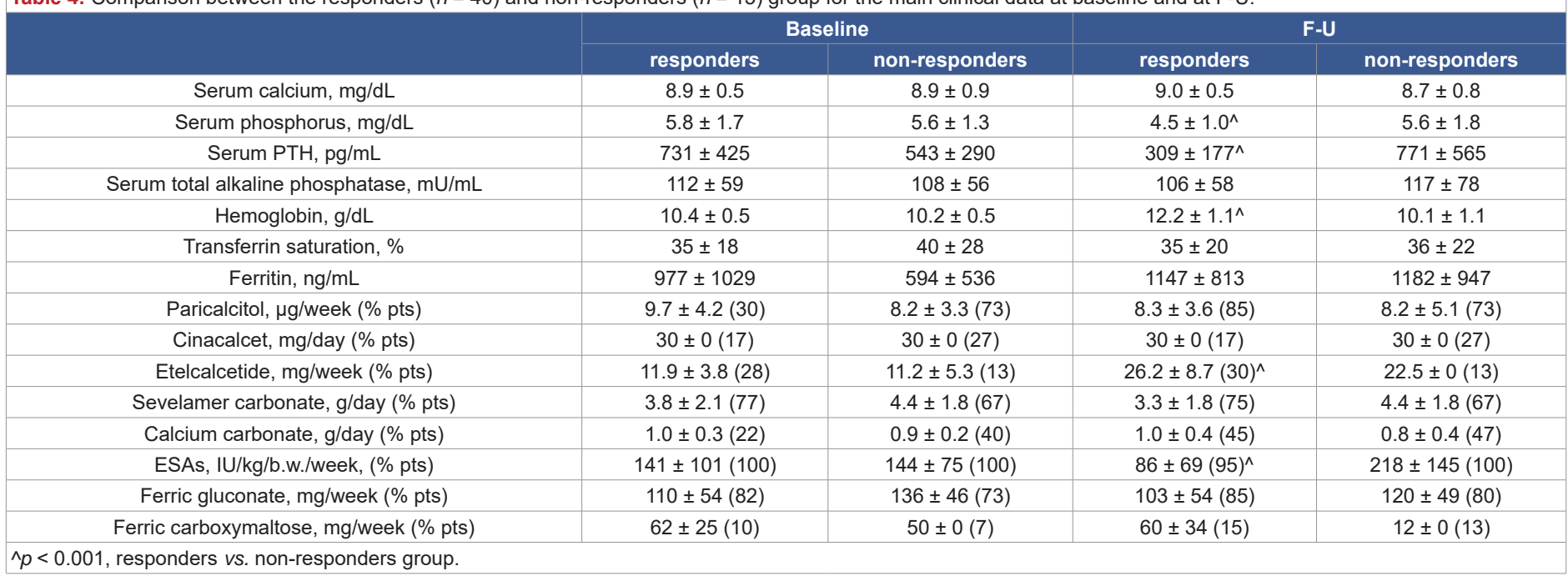

\section{$\square \mathrm{Hb} \leadsto \mathrm{PTH}$}

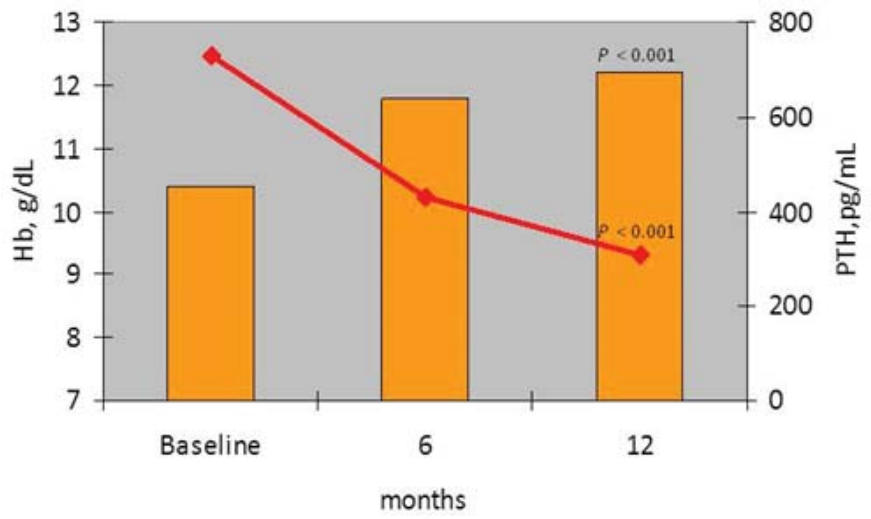

Figure 1: Trend in PTH Hb levels over the course of the study in the responders group ( $p=$ F-U vs. baseline)

significant correlations were found between serum PTH levels and serum phosphorous levels $(r=.328, p<0.001)$ and t-ALP levels ( $r=.450, p<0.001$ ). Further, ESAs doses were negatively correlated with TAST ( $r=-.216, p<0.001)$, and positively with ferritin levels $(r=.156, p<0.01)$, as well as PTH levels $(r=0.147, p<0.05)$. Total alkaline phosphatase was positively correlated with ferritin levels $(r=.278, p<$ $0.001)$. However, the multivariable adjusted linear regression model showed that the best and independent predictors of Hb levels were ESAs doses $(\beta=-0.315, p<0.0001)$ and PTH levels $(\beta=-0.262, p<0.001)$. At study completion, we overall observed a better control of SHPT. Indeed, a significant $27 \%$ and $15 \%$ reduction of PTH and phosphorous was noted.

Also, a significant increase of $\mathrm{Hb}$ values, without significant change in ESAs doses as well as a reduction in the number of patients treated with ESAs was also apparent (Table 1). At the same time, there were no significant change of TAST and ferritin levels, as well as for intravenous iron doses (Table 1). During the study period we did not record any hypocalcemic or hypercalcemic episodes who requiring calcimimetics or paricalcitol therapy adjustments. However, the reduction of serum calcium levels in patients treated with calcimimetics required a more frequent use of calcium carbonate (Table 1 ). Although at F-U there was an overall significant decrease in PTH levels, about 3 out of 4 patients (73\%) experience a PTH drop $\geq 30 \%$ (responders group) while the remaining $27 \%$ of the study cohort experienced a much lower reduction or an increase in PTH levels (non-responders group).

At study inception there were not statistically significant differences between the responders group $(n=40)$ and nonresponders group $(n=15)$, though the former group tended to have higher PTH levels ( $p>0.05$ ) (Table 4). However, significant differences in the biochemical characteristics between the 2 study groups were apparent at study completion (Table 4). In particular, among responders there was a mean $54 \pm 16 \%$ reduction in PTH levels, coupled with a significant increase in $\mathrm{Hb}$ levels, reduction in ESAs doses as well as a decrease in the number of patients treated with ESAs (Table 2). Notably, the increase in Hb levels was significant already after six months of SHPT treatment and was maintained throughout the study period (Table 2). To the contrary, a significant reduction in ESAs doses was appreciated only in the final part of the observation (Table 2). In the non-responders group $(n=15)$ the increase in PTH levels (+ $52 \pm 60 \%$ ), was not associated with a significant change in the Hb levels (Table 3). Similarly, a non-significant trend in ESA dose increase as well as no change in the number of patients treated with ESAs was also observed (Table 3). To further investigate the association of anemia and SHPT, we stratified responders according to treatment of SHPT. In particular, 14 subjects received a combination of calcimimetic and paricalcitol while the remainders $(n=26)$ received either vitamin D or calcimimetic as mono-therapy. Of note, subjects receiving a combination therapy exhibited significantly higher PTH levels than peers $(1118 \pm 529 \mathrm{pg} /$ $\mathrm{mL}$ vs. $544 \pm 178 \mathrm{pg} / \mathrm{mL}, p<0.001$ ) at study inception. At study completion, a similar PTH control was achieved and $\mathrm{Hb}$ 
levels increased significantly in both groups. Nevertheless, a statistically non-significant larger ESAs doses reduction was noted among patients receiving calcimimentic and vitamin D in combination. Lastly, in the responders group there were no significant differences in the $\mathrm{Hb}$ trend and in the ESAs doses between patients in single therapy with calcimimetic or paricalcalcitol.

\section{Discussion}

The present study aimed at evaluating whether adequate control of SHPT was coupled with an improvement in anemia control and a reduction in ESAs requirements in subjects receiving chronic HD. Indeed, ours results suggest that a reduction in PTH levels $\geq 30 \%$ from baseline by single or combination therapy with paricalcitol and calcimimetic in patients with $\mathrm{Hb}$ levels $<11 \mathrm{~g} / \mathrm{dL}$ on ESAs therapy is followed by an increase of $\mathrm{Hb}$ levels to values $>11 \mathrm{~g} / \mathrm{dL}$ and a concomitant reduction in ESAs usage. On the contrary, in patients who did not respond to treatment of SHPT serum PTH levels and ESAs requirement tented to increase while $\mathrm{Hb}$ levels did not change. These findings corroborate the notion that elevated serum PTH levels contribute to renal anemia and hyporesponsiveness to ESAs treatment, fueling the debate on the importance of SHPT control important in HD patients.

Secondary hyperparathyroidism (SHPT) is a common complication in HD patients [17].

Clinically, SHPT is associated with vascular and valvular calcification, renal osteodystrophy, and increased risk of cardiovascular and all-cause mortality [18-21]. Among the numerous complications of SHPT, anemia is rarely mentioned. Indeed, the KDIGO Clinical Practice Guidelines for Anemia management in Chronic Kidney Disease do not include serum PTH levels as a factor to be investigated in anemic subjects receiving dialysis [16]. Nevertheless, preliminary results support the notion that pharmacological and surgical treatment of SHPT is associated with an improvement in $\mathrm{Hb}$ levels and a reduction in ESAs requirement [10-13]. Differently from others, our study is characterized by precise selection criteria that allowed recruitment of a homogeneous cohort of patients on maintenance dialysis with the simultaneous presence of SHPT and anemia requiring ESAs therapy $[14,16]$. Furthermore, rather than focusing on a single therapy of for SHPT our study includes patients receiving different schemes of paricalcitol and/ or calcimimetics as mono-therapy or combination therapy [10-12]. Regardless of these methodological considerations, current results are consistent with previous reports and expand the body of evidence showing a significant increase in $\mathrm{Hb}$ levels six months after enforcement of PTH control below $300 \mathrm{pg} / \mathrm{ml}$. This effect may be attributed to the reduced toxic effects of PTH on the hematopoietic system [6,7]. As observed in a previous study [12], in our case series PTH levels were negatively correlated with $\mathrm{Hb}$ levels at baseline and a larger reduction of PTH was associated with a greater increase of $\mathrm{Hb}$ at follow-up. In line with what reported by others [10], the effect of SHPT treatment on Hb levels was only evident in the responders group and not observed in the non-responders group. Nevertheless, in our study cohort adequate control of SHPT (i.e. PTH $<300 \mathrm{pg} / \mathrm{ml}$ ) was also associated with a tendency in ESAs doses reduction which reached statistical significance among responders. In particular, the higher the PTH reduction was achieved during F-U the lower the ESAs requirement was needed to maintain adequate $\mathrm{Hb}$ levels at study completion. Finally, among responders the increase in $\mathrm{Hb}$ levels was recorded after only six months while the ESAs reduction occurred only after 12 months of strict PTH control, suggesting a time sequence of these events. Hence, although causal link cannot be inferred from these data, our observations lend credibility to the hypothesis of a close relationship between high PTH levels and anemia. A two-step hypothesis can be formulated to explain current findings. First, the reduction in PTH levels lead to an attenuation of the toxic effects of PTH on the hematopoietic system, in the short-term. Secondly, the reduction in PTH levels could lead to an improvement of the erythropoietic action of ESAs in the long-term through the reduction of bone marrow fibrosis. In fact, SHPT is one of the main conditions responsible for ESAs hyporesponsiveness [4]. Rao et al. showed that a greater degree of bone marrow fibrosis and higher PTH levels in patients with a poor response to ESAs compared to patients with a good response to ESAs [8]. Trunzo et al. found a significant reduction in weekly ESAs need 12 months after parathyroidectomy in nearly $65 \%$ of patients undergoing surgery [13]. Of interest, as hypothesized by authors the reduction of ESAs doses after parathyroidectomy is likely multi-factorial [22,23]. In particular, there could be a greater bio-availability of endogenous EPO and an improvement in sensitivity to the exogenous form [22, 23]. Although there are currently no studies that have evaluated the impact of parathyroidectomy or pharmacological treatments of SHPT on the evolution of bone marrow fibrosis, it is a widely shared opinion that the severity of SHPT and the extent of bone marrow fibrosis increase the ESAs doses required to maintain an adequate $\mathrm{Hb}$ response [24]. In these regards, a case of severe SHPT requiring monthly blood transfusions, despite the use of ESAs was described [25]. Of interest, bone marrow fibrosis was shown by bone biopsy [25]. The patient underwent parathyroidectomy and six months after surgery a repeated bone biopsy documented a complete resolution of bone marrow fibrosis [25]. Consistently, the late reduction in the need for ESAs observed in our cohort could be explained by a reduction in bone marrow fibrosis and increase in ESAs sensitivity due to a better control of SHPT. Alternatively a better SHPT control may be associated with a greater availability of endogenous EPO [8]. Although it was outside the scope of the current investigation, a differential impact of various SHPT agents on anemia have been postulated and 
several studies have reported an increase in Hb levels and/ or a reduction in the ESAs doses after treatment of SHPT with both VDRAs and calcimimetics [10-12]. Fusaro, et al. evaluated the impact of cinacalcet and VDRAs on the need for ESAs therapy in HD patients [26].

The study, conducted in a small number of patients, showed a greater efficacy of cincalcet compared to VDRAs in reducing the need for therapy with ESAs. Although indirect mechanisms cannot be excluded, these results seem to be attributable to a greater reduction in PTH levels rather than to a direct action of cinacalcet. However, calcimimetics rather than VDRAs can reduce Fibrobast Growth Factor 23 (FGF23) levels $[27,28]$. In turn, experimental data showed that FGF23 can suppress EPO production and expression of EPO receptor [9], lending credibility to clinical data which document a significant association between elevated levels of FGF23 and anemia in patients with mild to severe chronic kidney disease [29]. Hence, it cannot be excluded that the reduction in ESAs doses during cinacalcet therapy may be partly mediated by the reduction in FGF23 levels or other factors involved in the erythropoiesis process. However, in our study, we fail to show any significant differences regarding the impact of calcimimetics or VDRAs on Hb levels and ESAs doses among responders. Although the limited sample size may account for it, this last finding may also suggest that PTH reduction is the most important factor associated with anemia control.

Our experience has several limitations such as the retrospective, non-randomized study design and the limited number of patients enrolled. However, in the pre-post study design in which each patient is case and control as well as selection of a homogeneous study cohort through the implementation of inclusion and exclusion criteria allow for controlling for potential confounders. Indeed, while a causal link between SHPT and anemia cannot be inferred from these data, our findings further corroborate the hypothesis of a close relationship between high PTH levels and anemia. The adequate period of observation as well as the implementation of in-center clinical guidelines to homogenously monitor and correct iron deficiency are, in the authors' opinion, strengths of this observational study.

\section{Conclusion}

The present study showed that adequate control of SHPT is followed by an improvement of anemia and a reduction in the mean doses of ESAs. These results are likely due to the significant reduction in PTH levels, suggesting the important role of PTH in conditioning anemia and resistance to ESAs action. While future endeavors should test whether PTH reduction is causally linked to anemia improvement, our findings support PTH assessment as a potentially modifiable factor in anemic patients receiving maintenance dialysis. Indeed, the improvement of anemia and the reduction of ESA doses can lead to an improvement of the quality of life and survival as well as a reduction in costs connected with HD management.

\section{Acknowledgement}

All authors contributed to the conception or design of the work; or the acquisition, analysis, or interpretation of data; or have drafted the work or substantively revised it; and has approved the submitted version.

\section{References}

1. Foley RN, Parfrey PS, Harnett JD, Kent GM, Murray DC, et al. The impact of anemia on cardiomyopathy, morbidity, and mortality in end stage renal disease. Am J Kidney Dis. 1998; 31: 53-61. PubMed: https://pubmed.ncbi.nlm.nih.gov/8712222/

2. Eschbach, JW, Adamson J. Modern aspects of the pathophysiology of renal anaemia. Contrib Nephrol. 1988; 66: 63-70.

PubMed: https://pubmed.ncbi.nlm.nih.gov/3292149/

3. Eschbach JW, Egrie JC, Downing MR, Browne JK, Adamson JW. Correction of the anemia of end-stage renal disease with recombinant human erythropoietin. Results of a combined phase I and II clinical trial. N Engl J Med. 1987; 316:73-78.

PubMed: https://pubmed.ncbi.nlm.nih.gov/3537801/

4. Johnson DW, Pollock CA, Macdougall IC. Erythropoiesis-stimulating agent hyporesponsiveness. Nephrology (Carlton) 2007; 12: 321-330. PubMed: https://pubmed.ncbi.nlm.nih.gov/17635745/

5. Richardson D. Clinical factors influencing sensitivity and response to epoetin. Nephrol Dial Transplant. 2002; 17: 53-59. PubMed: https://pubmed.ncbi.nlm.nih.gov/11812914/

6. UrenaP,EckardtKU,SarfatiE,ZingraffJ,ZinsB,etal.Serumerythropoietin and erythropoiesis in primary and secondary hyperparathyroidism: Effect of parathyroidectomy. Nephron. 1991; 59: 384-393. PubMed: https://pubmed.ncbi.nlm.nih.gov/1758526/

7. Bogin E, Massry SG, Levi J, Djaldeti M, Bristol G, et al. Effect of parathyroid hormone on osmotic fragility of human erythrocytes. J Clin Invest. 1982; 69: 1017-1025.

PubMed: https://pubmed.ncbi.nlm.nih.gov/6281309/

8. Rao DS, Shih MS, Mohini R. Effect of serum parathyroid hormone and bone marrow fibrosis on the response to erythropoietin in uremia. N Engl J Med. 1993; 328: 171-175.

PubMed: https://pubmed.ncbi.nlm.nih.gov/8417383/

9. Coe LM, Madathil SV, Casu C, Lanske B, Rivella S, et al. FGF-23 is a negative regulator of prenatal and postnatal erythropoiesis. J Biol Chem 2014; 289: 9795-9810.

PubMed: https://pubmed.ncbi.nlm.nih.gov/24509850/

10. Goicoechea M, Vazquez MI, Ruiz MA, Gomez-Campdera F, PerezGarcía R, et al. Intravenous calcitriol improves anaemia and reduces the need for erythropoietin in haemodialysis patients. Nephron. 1998, 78, 23-27.

PubMed: https://pubmed.ncbi.nIm.nih.gov/9453399/

11. Mpio I, Boumendjel N, Karaaslan H, Arkouche W, Lenz A, et al Secondary hyperparathyroidism and anemia. Effects of a calcimimetic on the control of anemia in chronic hemodialysed patients. Pilot Study]. Nephrol Ther. 2011; 7: 229-236.

PubMed: https://pubmed.ncbi.nlm.nih.gov/21353659/

12. Tanaka M, Yoshida K, Fukuma S, Ito K, Matsushita K, et al. Effects of Secondary Hyperparathyroidism Treatment on Improvement in Anemia: Results from the MBD-5D Study. PLoS ONE. 2016; 20: 11: e0164865.

PubMed: https://pubmed.ncbi.nlm.nih.gov/27764168/

13. Trunzo JA, McHenry CR, Schulak JA, Wilhelm SC. Effect of parathyroidectomyonanemiaanderythropoietindosinginendstagerenal 
disease patientswith hyperparathyroidism.Surgery.2008;144:915-918. PubMed: https://pubmed.ncbi.nlm.nih.gov/19040997/

14. National Kidney Foundation. K/DOQI clinical practice guidelines for bone metabolism and disease in chronic kidney disease. Am J Kidney Dis. 2003; 42: S1-201.

PubMed: https://pubmed.ncbi.nlm.nih.gov/14520607/

15. Kidney Disease: Improving Global Outcomes (KDIGO)CKD-MBD Work Group. KDIGO clinical practice guideline for the diagnosis, evaluation, prevention, and treatment of chronic kidney disease-mineral and bone disorder (CKD-MBD). Kidney Int Suppl. 2009; 113: S1-S130. PubMed: https://pubmed.ncbi.nlm.nih.gov/19644521/

16. Kidney Disease: Improving Global Outcomes (KDIGO) Anemia Work Group. KDIGO Clinical Practice Guideline for Anemia in Chronic Kidney Disease. Kidney Int. Suppl. 2012; 2: 279-335.

17. Kidney Disease: Improving Global Outcomes (KDIGO) CKD-MBD Update Work Group. KDIGO 2017 Clinical Practice Guideline Update for the Diagnosis, Evaluation, Prevention, and Treatment of Chronic Kidney Disease-Mineral and Bone Disorder (CKD-MBD). Kidney Int Suppl. 2017; 7: 1-59.

PubMed: https://pubmed.ncbi.nlm.nih.gov/30675420/

18. Moe S, Drüeke T, Cunningham J, Goodman W, Martin K, et al. Kidney Disease: Improving Global Outcomes (KDIGO). Definition, evaluation, and classification of renal osteodystrophy: a position statement from Kidney Disease: Improving Global Outcomes (KDIGO). Kidney Int. 2006; 69: 1945-1953.

PubMed: https://pubmed.ncbi.nlm.nih.gov/16641930/

19. Nickolas TL, Leonard MB, Shane E. Chronic kidney disease and bone fracture: a growing concern. Kidney Int. 2008; 74: 721-731.

PubMed: https://pubmed.ncbi.nlm.nih.gov/18563052/

20. Hruska KA, Choi ET, Memon I, Davis TK, Mathew S. Cardiovascular risk in chronic kidney disease (CKD): the CKD-mineral bone disorder (CKD-MBD). Pediatr Nephrol. 2010; 25: 769-778.

PubMed: https://pubmed.ncbi.nlm.nih.gov/19898875/

21. Block GA, Klassen PS, Lazarus JM, Ofsthun N, Lowrie EG, et al. Mineral metabolism, mortality, and morbidity in maintenance hemodialysis. J Am Soc Nephrol. 2004; 15: 2208-2218.

PubMed: https://pubmed.ncbi.nlm.nih.gov/15284307/
22. Yasunaga C, Matsuo K, Yanagida T, Matsuo S, Nakamoto M, et al. Early effects of parathyroidectomy on erythropoietin production in secondary hyperparathyroidism. Am J Surg. 2002; 183: 199-204. PubMed: https://pubmed.ncbi.nlm.nih.gov/11918889/

23. Rault R, Magnone M. The effect of parathyroidectomy on hematocrit and erythropoietin dose in patients on hemodialysis. ASAIO J. 1996; 42: M901-903.

PubMed: https://pubmed.ncbi.nlm.nih.gov/8945016/

24. Tanaka M, Komaba H, Fukagawa M. Emerging Association Between Parathyroid Hormone and Anemia in Hemodialysis Patients. Ther Apher Dial. 2018; 22: 242-245.

PubMed: https://pubmed.ncbi.nlm.nih.gov/29767854/

25. Azevedo PDGG, Cardoso PSR, Farah KP, de Melo FHC, Rezende SM. Complete reversal of bone marrow fibrosis after parathyroidectomy for secondary hyperparathyroidism. Br J Haematol. 2017; 178: 500. PubMed: https://pubmed.ncbi.nlm.nih.gov/28556955/

26. Fusaro M, D'Angelo A, Naso A, Frigo AC, Miozzo D, et al. Treatment with calcimimetic (cinacalcet) alters epoetin dosage requirements in dialysis patients: preliminary report. Ren Fail. 2011; 33: 732-735. PubMed: https://pubmed.ncbi.nlm.nih.gov/21671845/

27. Moe SM, Chertow GM, Parfrey PS, Kubo Y, Block GA, et al. Evaluation of Cinacalcet $\mathrm{HCl}$ Therapy to Lower Cardiovascular Events (EVOLVE) Trial Investigators . Cinacalcet, fibroblast growth factor-23, and cardiovascular disease in hemodialysis: The Evaluation of Cinacalcet $\mathrm{HCl}$ Therapy to Lower Cardiovascular Events (EVOLVE) trial. Circulation. 2015; 132, 27-39.

PubMed: https://pubmed.ncbi.nlm.nih.gov/26059012/

28. Wolf M, Block GA, Chertow GM, Cooper K, Fouqueray B, et al. Effects of etelcalcetide on fibroblast growth factor 23 in patients with secondary hyperparathyroidism receiving hemodialysis. Clin Kidney J. 2019; 13: 75-84.

PubMed: https://pubmed.ncbi.nlm.nih.gov/32082556/

29. Mehta R, Cai X, Hodakowski A, Lee J, Leonard M, et al. Fibroblast growth factor 23 and anemia in the chronic renal insufficiency cohort study. Clin J Am Soc Nephrol. 2017; 12: 1795-1803.

PubMed: https://pubmed.ncbi.nlm.nih.gov/28784656/ 